\title{
Implementation of point-of-care blood gas testing at a large community hospital: Cost analysis, sepsis bundle compliance, and employee engagement
}

\author{
Robert A. Chase $M D^{1}$, Patricia A. DeJuilio MS, RRT'1 , Jeffrey P. Huml MD ${ }^{1}$, Alex P. Johnson APN ${ }^{1}$, Ryan M. Kaminski RRT ${ }^{1}$
}

\begin{abstract}
RA Chase, PA DeJuilio, JP Huml, AP Johnson, RM Kaminski. Implementation of point-of-care blood gas testing at a large community hospital: Cost analysis, sepsis bundle compliance, and employee engagement. Can J Respir Ther 2021;57:60-67. doi: 10.29390/cjrt-2021-008.

Introduction/Background: Point-of-care testing (POCT) platforms support patient-centered approaches to health care delivery and may improve patient care. We evaluated implementation of a POCT platform at a large, acute care hospital in the Midwestern United States.

Methods: We used lactate testing as part of a sepsis bundle protocol to evaluate compliance and mortality outcomes. Respiratory team members were surveyed to assess perception of efficiency, ease of use, timely patient care, and overall engagement with the POCT system. Annualized cost per test of a benchtop analyzer and a POCT platform were compared across 3 years for each platform.

Results: Lactate testing volume increased from $61 \%$ to $91 \%$, which was associated with improved sepsis bundle protocol compliance. Employees reported high levels of engagement, improvements in efficiency and time savings, and better patient care with POCT. Average cost per test was $\$ 10.02$ for the benchtop system and $\$ 6.21$ for the POCT platform. POCT saved our institution $\$ 88,476$ annually in labor costs.

Discussion: Combined with a robust training program emphasizing the use of lactate testing in the context of the overall clinical picture, POCT enabled adherence to the sepsis bundle protocol and may have contributed to lower mortality. Additionally, the COVID-19 pandemic has provided us with unanticipated benefits of using POCT; it has enhanced our ability to deal with stringent infectious disease protocols, saving time and minimizing patient and staff exposure. Conclusions: Implementation of a POCT platform was associated with improved compliance to our sepsis protocol, reduced sepsis mortality, high employee engagement, and cost savings.
\end{abstract}

Key Words: respiratory therapy; patient-centered care; process improvement; lactate; lab test; COVID-19

\section{INTRODUCTION}

Real-time diagnostic test results for clinician decision-making can improve patient outcomes and be cost-effective [1-6]. In addition to published data that support the value of point-of-care testing (POCT), which is performed at or near the patient's bedside and provides real-time results [7], hospital administrators want to know how the implementation of POCT at their unique institutions will affect quality and costs. Hospitals tend to adopt POCT for clinical assays that have been shown to be accurate and add value by improving clinical outcomes while reducing cost or time to result [1].

Reduced length of stay and a subsequent decrease in associated health care cost may be associated with POCT. Results of studies conducted in emergency departments suggest reduced length of stay in the Emergency Department and decreased hospital admission for patients in whom POCT was used compared to patients served by central laboratory testing [4, 8-11]. Implementation of POCT has been recommended for hospitals examining ways to improve hospital efficiency and reduce cost [12]. These reductions, based on obtaining POCT results faster than central laboratory results, need not sacrifice patient-centered approaches that support and improve patient care in hospitals [13]. As an example, time critical changes to care management have been observed for $7 \%$ of patients and unnecessary treatment prevented for nearly $4 \%$ of 1728 patients enrolled in a randomized controlled trial in the United Kingdom [14].
Our institution is a $400+$ bed acute care hospital in the US Midwest. Designated as a stroke center, it has 46 adult intensive care unit (ICU) beds, a PICU/Pediatric Emergency Department, and a Level III NICU. The Respiratory Care Department is responsible for analyzing all arterial blood gas samples in the hospital, which are rapid indicators of tissue oxygenation, adequate ventilation, and acid-base disturbances. They are relied upon frequently in emergency departments and critical care situations to quickly determine possible causes of acute onset of difficulty breathing, shortness of breath, and altered mental status [15, 16].

In 2011, we began the process of evaluating new benchtop and POCT blood gas analyzers to replace the aging traditional bench top systems, which were strategically located in four satellite blood gas laboratories throughout the hospital. The hospital had approved the capital necessary for the purchase (Fiscal Year 2012, beginning 1 July 2011). In August 2011, a team from our hospital was formed to review the options for the new blood gas analyzers and make a recommendation for the final purchase. This team consisted of respiratory and physician leads, laboratory experts, and hospital administrators. Four analyzer models were chosen for evaluation, including two benchtop and two POCT models. Clinical, operational, and financial criteria were evaluated for each of the analyzers under consideration, and in October 2011, the decision was made to purchase one of the POCT platforms. During implementation and training on the new analyzers, which occurred in

${ }^{1}$ Northwestern Medicine Central DuPage Hospital, Winfield, IL, USA

Correspondence: Patricia A. DeJuilio, MS, RRT, Clinical Director Respiratory Care and Sleep Center, Northwestern Medicine Central DuPage Hospital, 25 N. Winfield Rd., Winfield, IL 60190 USA. 
November and December 2011, both the old benchtop analyzers and the new POCT devices were used for testing. All blood gas testing was transitioned to the POCT platform in January 2012.

During our evaluation of the new blood gas systems, the performance of lactate testing in sepsis diagnosis emerged as an important metric at our institution. There are an estimated 1.7 million adult sepsis cases annually and 265,000 deaths reported nationally, and in 2013, sepsis cost the United States' health care system 24 billion dollars annually [17-19]. Serum lactate has been shown to help clinicians diagnose, risk-stratify, and treat patients with sepsis, and as a reliable predictor of outcome, it can provide the earliest clue of cardiovascular compromise and poor tissue perfusion that indicate treatment progress [20-24]. Our institution has had care pathways for sepsis in place for some time; however, we implemented the revised Sepsis Care Bundle (Surviving Sepsis Campaign (SSC), 2012) in advance of purchasing the new blood gas system $[21,25]$. The sepsis protocol aimed for completion of four actions within a 3 -h window for patients suspected with sepsis: measure lactate level, obtain blood cultures prior to administration of antibiotics, administer broad spectrum antibiotics, and administer $30 \mathrm{~mL} / \mathrm{kg}$ crystalloid for hypotension or lactate values at least $4 \mathrm{mmol} / \mathrm{L}$. We recognized that it was difficult to consistently predict the timing from a clinician suspecting sepsis and entering a lactate test order, to the clinician receiving the test result from the central laboratory, which could delay clinical decision-making. Following transition to the new POCT platform in 2012, to reduce the time from blood draw to results, the respiratory therapists began running lactate tests on the POCT in response to sepsis alerts, and bundle compliance was closely monitored.

We report on the results of our evaluation process of a POCT blood gas analyzer and the use of this platform in a quality improvement initiative for sepsis bundle compliance using lactate testing, and comment on its relevance today, during the COVID-19 pandemic. To our knowledge, there has not been a comprehensive study to look at outcomes of implementation of blood gas POCT by a respiratory care department [26]. Our objective was three-fold in this retrospective analysis. We aimed to evaluate sepsis bundle compliance and patient outcomes, employee engagement responses to POCT blood gas analyzers, and the cost of blood gas analysis before and after implementation of POCT testing, comparing to the benchtop analyzer it replaced. We hypothesized that implementation of POCT for blood gases would reduce hospital cost and improve engagement among the respiratory care team, and the use of POCT lactate testing would be associated with improved patient outcomes. Such a result could help hospitals reduce economic burdens while performing safely and reliably, and could help alleviate the added challenges posed by infection prevention protocols in use during the current COVID-19 pandemic [7, 27].

\section{Data sources and equipment}

\section{METHODS}

Retrospective data were extracted from our institution's electronic medical record system (EPIC, Verona, WI, USA). No patient personal health information was extracted, no data included personal identifiers, and survey data were collected anonymously in this process improvement initiative, therefore our institutional review board did not require review.

The four existing benchtop blood gas analyzers were manufactured by Radiometer America, Inc. (Brea, CA, USA). The POCT system we evaluated was Epoc (Siemens Medical Solutions USA, Inc., Malvern, PA).

\section{Evaluation period}

We evaluated blood gas testing data between 2009 and 2014. Because our analyses were retrospective, each analysis comprised data from slightly different sampling periods during this time. Sepsis bundle compliance was evaluated from 2012 through 2015. Mortality due to sepsis was evaluated from January 2014 through February 2015. Employee engagement was evaluated only for team members who had experience with both the benchtop analyzers and the POCT, thus surveys were distributed in January 2019. Our cost analysis evaluation for the POCT was conducted from 2012 to 2014 and was compared to costs for the bench analyzers collected from 2009 to 2011. Because both analyzers were used during a portion of the implementation, we excluded October 2011 through February 2012 from the cost analysis.

\section{Sepsis bundle compliance}

We assessed improvement in our care process by measuring compliance with our sepsis bundle. Lactate testing compliance was measured as the percentage of patients who had lactate testing completed within $6 \mathrm{~h}$ of first meeting severe sepsis or septic shock criteria divided by the total number of patients meeting criteria for severe sepsis or septic shock. We evaluated this parameter by comparing lactate compliance rates from the first three quarters following implementation of the POCT system to baseline lactate compliance using the central lab in 2012. We graphically compared lactate compliance to benchmarks published as part of the SSC, in which lactate compliance was $61 \%$ in the first quarter tested and rose to $78.7 \%$ compliance during a 2 -year study period [25].

Because lactate is part of a bundle of practices, and subsequent actions for treating sepsis cannot occur without its result, we also evaluated overall compliance to the Sepsis Bundle Protocol. We monitored the percentage of patients suspected of sepsis for whom the following were completed within a 3-h window: lactate levels and blood cultures obtained (blood cultures taken prior to administration of antibiotics), administration of broad-spectrum antibiotics, and in the case of hypotension or lactate of at least $4 \mathrm{mmol} / \mathrm{L}$, administration of $30 \mathrm{~mL} / \mathrm{kg}$ crystalloid.

Sepsis mortality was used to assess patient outcomes as an indicator of the quality of compliance to the Sepsis Bundle Protocol. The percent mortality observed in patients diagnosed with sepsis was calculated monthly from January 2014 through February 2015 using the number of all-cause deaths in patients with a diagnosis of sepsis divided by the total number of patients with a diagnosis of sepsis. These rates were compared graphically to results published from the SSC that showed a decrease in unadjusted hospital mortality rates from $37 \%$ to $30.8 \%$ following implementation of a sepsis bundle protocol [25].

\section{Employee engagement}

We distributed an anonymous survey to the respiratory therapists during mandatory staff meetings after the POCT was implemented. Team members were asked to complete the surveys only if they were part of the team when the benchtop analyzers were in use. The surveys assessed staff perception of efficiency, ease of use, timely patient care, and overall satisfaction with the use of point-of-care testing.

\section{Cost of blood gas testing}

We calculated average monthly total cost-per-test for the benchtop test from January 2009 through September 2011 prior to POCT implementation and from March 2012 through December 2014 for the POCT system, excluding months during which both analyzers were in use. Parameters measured for both devices included sample device and materials, quality control material, maintenance agreement, and cost of the device itself. The blood gas cartridge that we utilize contains each of the analytes we measure including lactate; there is no additional cost for specific analytes. The point-of-care device cost included an additional analyzer that would run methemoglobin and carboxyhemoglobin. We estimated the time saved by the technician per test using the results of the employee engagement survey.

\section{Sepsis bundle compliance}

\section{RESULTS}

Lactate testing compliance at baseline was 61\% annually in 2012, which was similar to the SSC results [25]. Following implementation of the POCT testing the annual rate of testing in 2015 was $91 \%$, which exceeded SSC results of $78.7 \%$ compliance achieved (Figure 1). The rates of compliance with the sepsis bundle protocol increased from the baseline period to the post-implementation period, reflecting a similar trend as lactate testing. During our evaluation, monthly mortality rates in sepsis patients decreased from $41.7 \%$ at the beginning of our assessment (January 2014) to 8\% in February 2015 (Figure 2), showing a larger reduction in mortality than results from the SSC study. 


\section{Chase et al.}

\section{FIGURE 1}

Sepsis bundle.

$C D H=$ Central DuPage Hospital; ICU = Intensive Care Unit; $E D=$ Emergency Department.

\section{Sepsis Bundle}

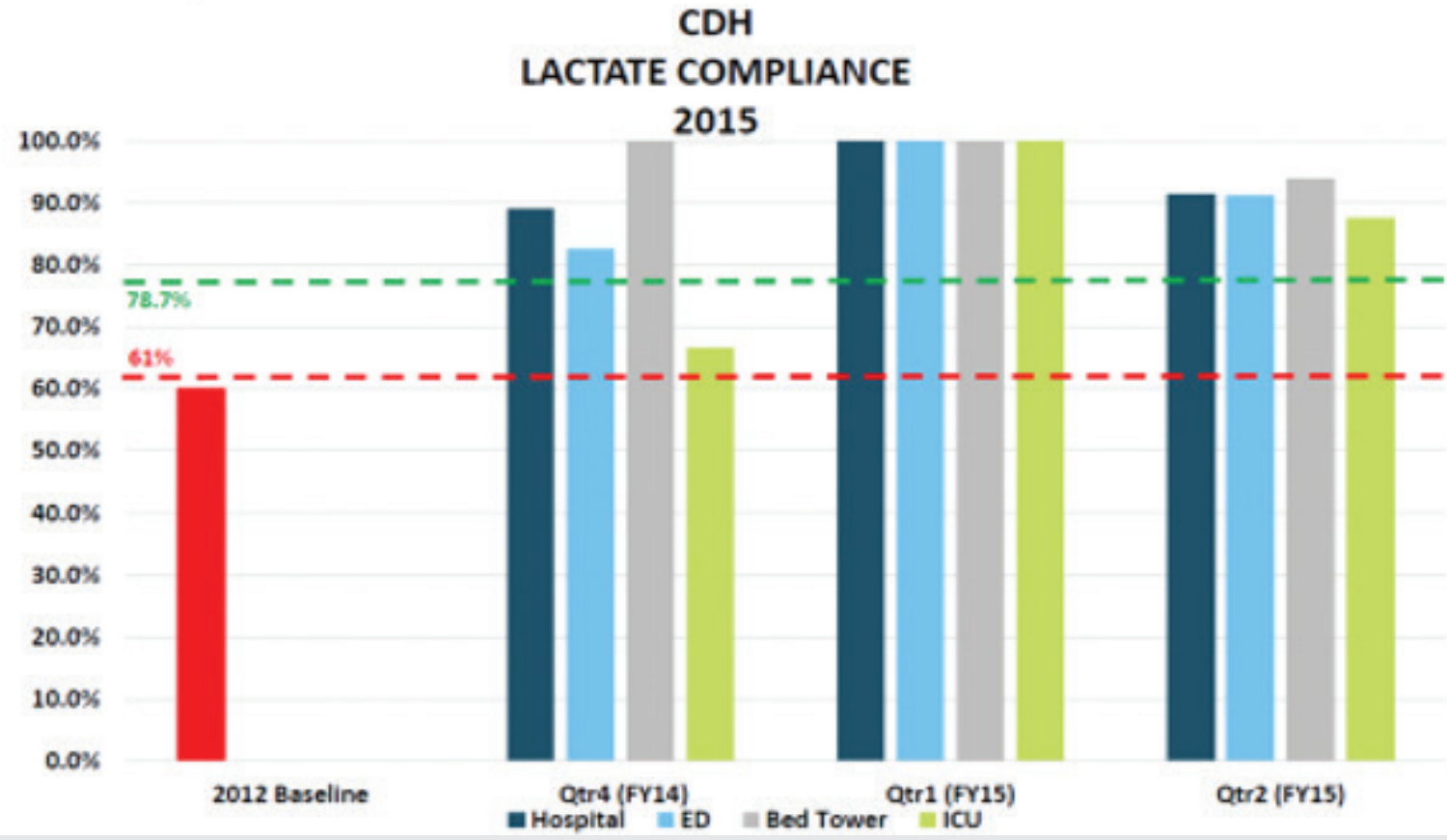

FIGURE 2

OVERALL SEVERE SEPSIS OBSERVED MORTALITY 2014-2015.

$\mathrm{CDH}=$ Central DuPage Hospital.

Overall Severe Sepsis Observed Mortality 2014-2015

Sepsis Bundle

OVERALL SEVERE SEPSIS OESERVED MORTALITY

2014/2015

coss

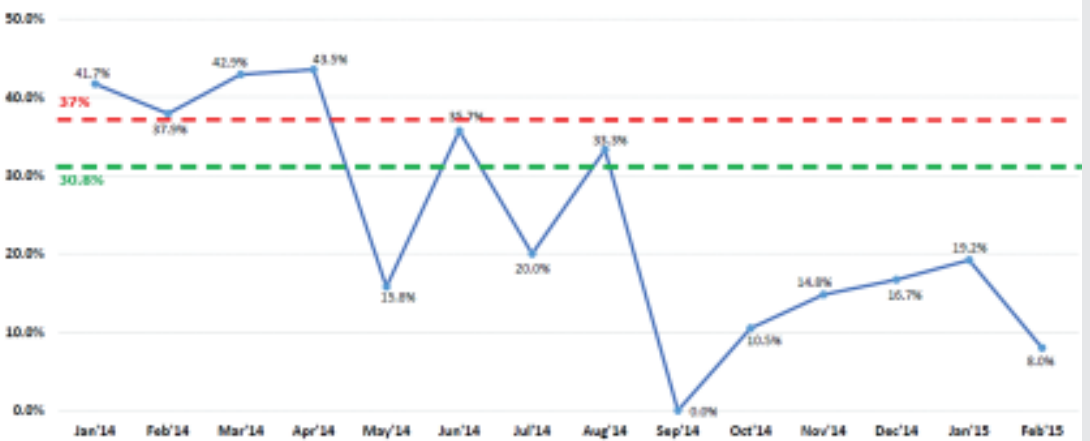




\section{Employee engagement}

Out of the 64 respiratory team members, paper surveys were distributed to 62 members who attended the staff meetings where they were handed out. Among these, 33 members had experience with both the benchtop analyzers and point of care devices. All 33 (100\%) team members with this experience completed the survey. Most of the respondents (81\%) indicated that the POCT saved them at least $10 \mathrm{~min}$ of time per sample. Respondents were extremely satisfied with their experience with the POCT system and strongly agreed that the POCT system had improved their efficiency at high levels (73\%), 58\% thought it was extremely easy to use, and $85 \%$ believed it allowed them to deliver more timely care to patients (Table 1).

Other notable comments from the respondents were regarding workflow. Respondents found it beneficial to not have to leave a critical patient situation to run a blood gas sample but could remain with the patient and the critical care team. The new workflow allowed them to remain part of the critical care team, which they believed produced

TABLE 1

Respiratory care team experience with point-of-care blood gas analyzers

\begin{tabular}{llc}
\hline Survey questions & \multicolumn{2}{c}{$\begin{array}{c}\text { Responses, } \\
N=33 \text { respondents }\end{array}$} \\
\cline { 2 - 3 } & $n$ & $\%$ \\
\hline
\end{tabular}

1. Since $\mathrm{CDH}$ transitioned to $\mathrm{POC}$, do you believe the total time it

takes from obtaining to resulting a

blood gas compared to benchtop

analyzers is:

Same; time to result not changed

Save $5-10$ min per sample

Save 10-15 min per sample

Save $>15$ min per sample

2. Rate overall experience with $\mathrm{POC}$ blood

gas device.

Extremely satisfied

Somewhat satisfied

Made no difference

Fairly satisfied

Not at all satisfied

3. Agree or disagree with statement:

"I believe that POC has improved

my efficiency."

Strongly agree

Agree

Somewhat agree

Do not agree

4. On a scale of $1-5$, rate the POC use;

$1=$ hardest to use; 5 = extremely

easy to use.

1

2

3

4

5

5. Rate the Following: "I believe that POC

allows me to deliver more timely care to

my patients."

All the time

Some of the time

No difference in time

POC takes more time than benchtop

analyzers

6. Overall, rate how POC have affected

your job satisfaction.

Tremendously improved

Improved

Somewhat improved

Not improved

$\begin{array}{cc}0 & 0 \\ 11 & 33 \\ 6 & 18 \\ 16 & 48\end{array}$

$\begin{array}{cc}24 & 73 \\ 7 & 21 \\ 0 & 0 \\ 2 & 6 \\ 0 & 0\end{array}$

$24 \quad 73$

927

$0 \quad 0$

0

Note: $\mathrm{CDH}$, Central DuPage Hospital; POC, point-of-care.

more seamless patient care and improved their job satisfaction. Delays in care associated with the benchtop analyzers were noted by the survey respondents to include lost samples that required additional blood draw, hemolysis due to time between draw and injection into machine, difficulty locating physician due to time passed, and errors in transcribing results into electronic medical record.

\section{Cost of blood gas testing}

The average monthly blood gas testing volume in our hospital over the 3-year baseline evaluation was 829 tests on the benchtop analyzer (January 2009-September 2011) at an average monthly cost of $\$ 10.02$ per test (unadjusted for inflation). After POCT implementation, average monthly testing volume on just the POCT platform increased to 1298 tests across the 3-year evaluation period (March 2012-December 2014 ) with an average monthly price per test of $\$ 6.21$ (Table 2, Figure 3).

Volume of testing steadily increased each year, beginning in 2009 with 9977 tests for the year, and ending with 19,845 tests overall in 2014. Total average annual costs of testing were similar between the two platforms, excluding the months using both analyzers, and without considering labor was $\$ 91,409$ for the bench top analyzer and $\$ 91,339$ for the POCT system.

We factored in a labor cost savings based on results from the Employee Engagement Survey results described above. From that survey, we determined that a minimum of $10 \mathrm{~min}$ was saved in labor time for each test run on the POCT system. Based on an average monthly testing volume of 1298 tests across 34 months of evaluating the POCT, approximately $216 \mathrm{~h}$ per month were saved in respiratory therapist time. At an average hourly cost of labor (including direct and indirect costs) of $\$ 34.07$ per hour, an estimated $\$ 7373.04$ was saved monthly. This cost savings resulted in an annual cost savings estimated at $\$ 88,476$ when the reduced labor for POCT testing is considered (Table 3).

\section{DISCUSSION}

Our evaluation of a POCT blood gas testing platform, following implementation at our institution, found that it allowed for better compliance with lactate testing and sepsis bundle protocols, resulted in higher levels of employee engagement, and saved the hospital nearly $\$ 4$ per test over 3 years with a corresponding estimate of $\$ 88,000$ saved in labor costs, compared to the bench top analyzer system previously in use. Overall cost for testing equipment and supplies was similar between the two analyzer systems in the two evaluation periods; however, testing steadily increased each year, to the extent that nearly twice the tests were completed in 2014 than in 2009.

Financial and clinical benefits have been reported for facilities that utilize POCT. The speed in which a clinician receives an answer, diagnosis, and treats can be increased substantially with POCT. This may lead to a decrease in length of stay in a health care facility, which in turn reduces overall cost to patients and payers $[1,4,8,9]$. Initially, we viewed cost as a barrier to implementation of POCT blood gas; however, after careful review and analysis of current spending, we predicted that the transition to POCT may result in cost neutrality or possibly cost savings, a result that we saw bear out in our data. Studies have shown that POCT cost when compared to a central laboratory may be less, more, or may show no difference in cost, so it was important for us to evaluate POCT within our institution and systems [2-4, 8, 28, 29].

Patient survival improved as our institution increased lactate testing compliance and adherence to the sepsis bundle protocol. Although the methodology of our evaluation cannot prove that improved survival was caused by implementation of POCT, we observed increased testing and adherence to the sepsis protocol immediately following implementation of POCT and the systems we developed as part of that implementation. Trends in compliance and mortality rates at our institution trended similarly to those of the SSC study [25], but the magnitude of our improvements appear to have exceeded those reported in the SSC study. We believe POCT largely enabled the increased testing and compliance at our institution. Lactate monitoring can identify at-risk patients early and has been linked to survival of sepsis [30, 31], 
TABLE 2

Blood gas comparison including capital expense in US dollars

\begin{tabular}{|c|c|c|c|c|c|c|c|c|c|}
\hline & \multicolumn{6}{|c|}{ Annualized comparison } & \multicolumn{3}{|c|}{ Overall comparison } \\
\hline $\begin{array}{l}\text { Point of care } \\
\text { analyzer plus } \\
\text { CO-oximetry } \\
\text { analyzer }\end{array}$ & $\$ 92,232$ & $\$ 10,580$ & $\$ 4480$ & $\$ 107,292$ & 15,581 & $\$ 6.89$ & $\$ 321,875$ & $(265,429)$ & $(\$ 284,710)$ \\
\hline
\end{tabular}

${ }^{a}$ Annualized cartridge or test card cost. Includes quality control and calibration.

${ }^{\mathrm{b}}$ Annualized cost of investment and middleware.

${ }^{\mathrm{c} A n n u a l i z e d}$ service costs or contracts.

${ }^{\mathrm{d}}$ Total annualized cost.

${ }^{e}$ Annualized number of tests.

${ }^{\mathrm{f}}$ Annualized cost per test including capital costs.

${ }^{9}$ Total cost for each 3-year testing period.

${ }^{\mathrm{h}}$ Point of care 3-year cost savings.

'Point of care savings including testing and labor cost.

TABLE 3

Total cost of blood gas sampling per month for a 6-year period (in US dollars unadjusted for inflation)

\begin{tabular}{|c|c|c|c|c|c|c|}
\hline Month & 2009 & 2010 & 2011 & 2012 & 2013 & 2014 \\
\hline February & $\$ 2152$ & $\$ 1858$ & $\$ 4700$ & excluded & $\$ 10,169$ & $\$ 11,239$ \\
\hline March & $\$ 6800$ & $\$ 55,394$ & $\$ 10,631$ & $\$ 5293$ & $\$ 8433$ & $\$ 9373$ \\
\hline May & $\$ 1858$ & $\$ 3327$ & $\$ 9275$ & $\$ 3310$ & $\$ 6811$ & $\$ 10,068$ \\
\hline June & $\$ 10,394$ & $\$ 1982$ & $\$ 8736$ & $\$ 5299$ & $\$ 6683$ & $\$ 9178$ \\
\hline July & $\$ 6140$ & $\$ 11,175$ & $\$ 13,095$ & $\$ 3658$ & $\$ 6945$ & $\$ 11,161$ \\
\hline October & $\$ 5421$ & $\$ 4565$ & excluded & $\$ 4065$ & $\$ 9079$ & $\$ 11,713$ \\
\hline November & $\$ 5955$ & $\$ 4114$ & excluded & $\$ 7043$ & $\$ 7457$ & $\$ 12,441$ \\
\hline December & $\$ 2962$ & $\$ 6806$ & excluded & $\$ 5828$ & $\$ 7262$ & $\$ 10,446$ \\
\hline Total \$ per year* & $\$ 70,384$ & $\$ 134,641$ & $\$ 69,201$ & $\$ 43,710$ & $\$ 101,313$ & $\$ 128,993$ \\
\hline Average monthly $\$$ by year & $\$ 5865$ & $\$ 11,220$ & $\$ 7689$ & $\$ 4371$ & $\$ 8443$ & $\$ 10,749$ \\
\hline Average monthly $\$$ across 3 -year period & $\$ 8310$ & $\$ 8310$ & $\$ 8310$ & $\$ 8059$ & $\$ 8059$ & $\$ 8059$ \\
\hline Total \# tests per year & 9977 & 9269 & 10,829 & 11,098 & 15,054 & 19,845 \\
\hline $\begin{array}{l}\text { Average monthly \$ per test across each } \\
\text { 3-year period }\end{array}$ & $\$ 10.02$ & $\$ 10.02$ & $\$ 10.02$ & $\$ 6.21$ & $\$ 6.21$ & $\$ 6.21$ \\
\hline
\end{tabular}

*Total cost included all sampling devices, test cards, quality control reagents, and maintenance of analyzers.

so we were not surprised to see improved survival associated with increased testing. However, we also developed additional systems as part of the implementation of the POCT system. We undertook a substantial training program, under which all 64 team members were trained twice to be confident we were operating efficiently. Our sepsis program training reinforced that lactate was not a biomarker for sepsis and does not alone diagnosis sepsis. Lactate must be considered in context of the entire patient physiologic evaluation and clinical picture before it can be determined what the lactate value means for a particular patient. Additional guiding principles were implemented organizationally that enhanced the rigor of our sepsis program and encouraged lactate testing, including the following.

Using lactate as a prognostic indicator

In other words, the higher a lactate is in sepsis, the higher the risk of mortality. The converse is also true, that the lower a lactate value is in a sepsis patient, the greater the chance of survival [32].
Using lactate clearance as a metric for treatment efficacy

The faster the lactate clears, the better the response to treatment. Although lactate clearance can vary significantly in sepsis, decreasing values is generally a good prognostic sign [33].

Teaching team members to be aware of false elevations Patients with renal failure, liver failure, or patients taking metformin may also exhibit false elevations in blood lactate. This may be due to causes such as decreased lactate clearance or "Type B" lactic acidosis.

Using lactate as an identifier of "Cryptic Shock"

Cryptic shock is when critical hypoperfusion (lactate $>4 \mathrm{mmol} / \mathrm{L}$ ) exists in the setting of normal pressures. In a sepsis resuscitation study by Puskarich et al., it was found that patients with cryptic shock (lactate $>4 \mathrm{mmol} / \mathrm{L}$ (>36 mg/dL) with a blood pressure $>90 \mathrm{mmHg}$ ) had an observed mortality of $21 \%$, whereas patients with overt shock had a 


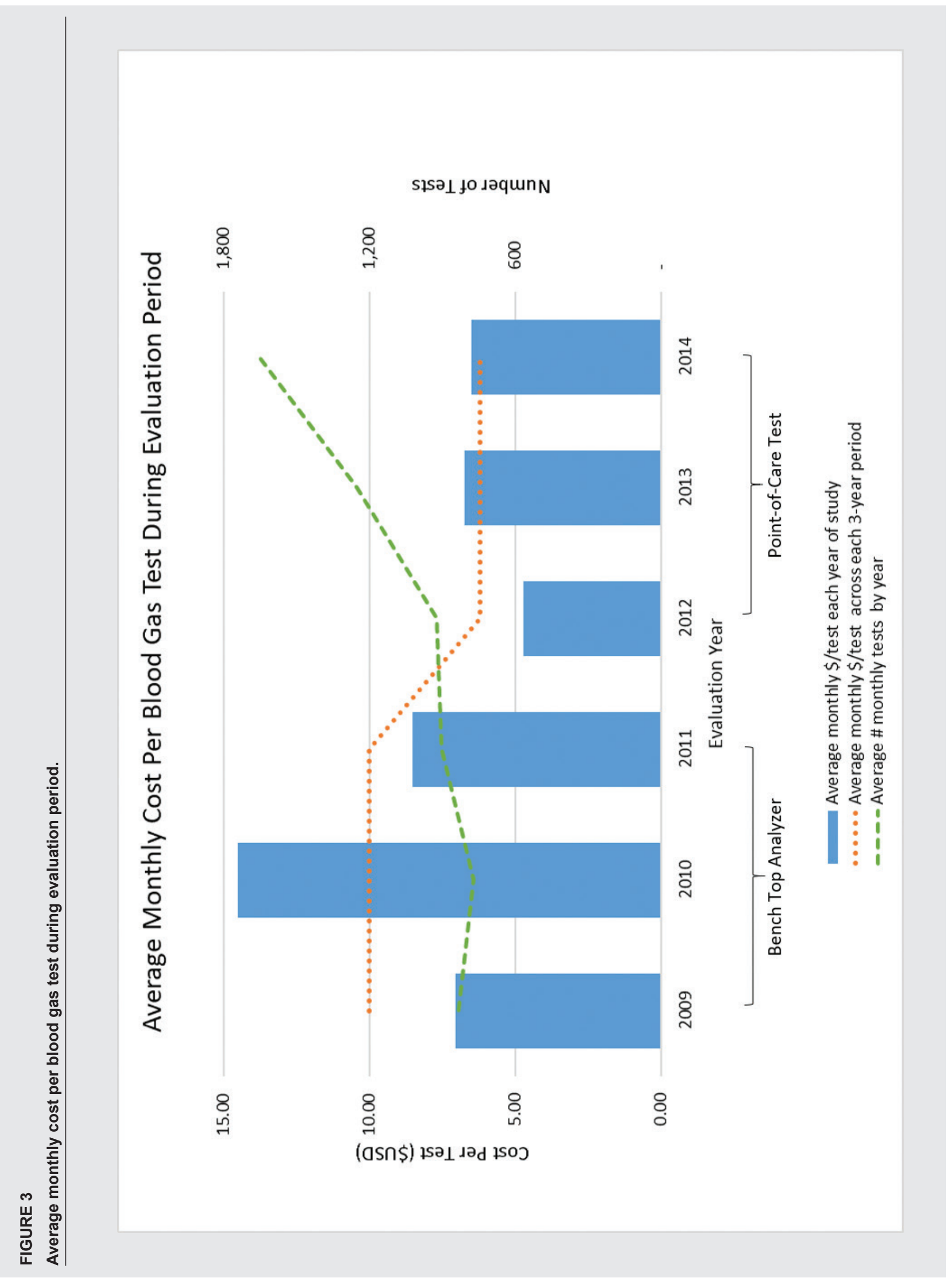


mortality of $19 \%$, suggesting that lactate may help identify at-risk patients earlier than conventional vital signs [34].

An addition of "lactate best practices" in the program included encouraging clinicians to ask whether an elevated lactate result could possibly be related to the source of infection. With this question, providers treating a sepsis patient were inherently acknowledging the numerous possible causes for an elevated lactate, and yet were still essentially asking, "Could it be sepsis?" This is because even if the index of suspicion of infection was low in the setting of lactate evaluation, the sepsis bundle was still implemented. This practice decreases the likelihood of overlooking patients with possible sepsis. With the help of rapid lactate results, patients were essentially "ruled in" for sepsis until they could be "ruled out." The team also established parameters for when to draw venous vs. arterial lactates, considerations for tourniquet use that can falsely elevate results, as well as an "always on" order set that enabled staff to acquire repeat lactates for trending when initial values were elevated.

Historically in our hospital, respiratory care practitioners obtained a sample of blood and physically transported the sample through patient care areas to bench top analyzers. Once a result was obtained, the sample data were printed out and manually entered into laboratory software, which interfaced with the electronic medical record; a process that took at least $10 \mathrm{~min}$. We hoped to decrease the load on our hospital central laboratory, which was experiencing increased testing volume and subsequent increased turnaround time. Additionally, we hoped that implementing the POCT system would mitigate delays in care from lost samples and the necessary replacement blood draw, hemolysis due to protracted times between collection and testing, difficulty locating physician for reporting due to lengthy elapsed times to obtain results, and errors in transcribing results into electronic medical record.

POCT had a number of positive impacts on workflow, as well as patient outcomes, due to workflow improvements. Immediate transfer of blood to the machine helps avoid the need for redraws. Barcode scanning and operator lockout features help improve accuracy of patient and sample identification and result matching because the POCT has positive patient identification features. The POCT uses Wi-Fi technology that directly imports data to the electronic medical record system, which eliminates the need for manual entry of results, adding convenience for clinicians [2]. Additionally, we have been surveyed twice by College of American Pathologists since switching to the POCT system and because we no longer require blood gas laboratories, the inspections were rather simple, and we have passed without recommendations. Routine side-by-side comparison to the central laboratory occurs and consistently demonstrates the accuracy of the POCT results.

The goal of most hospitals is to reduce economic burdens while performing safely and reliably [7]. The ability to provide immediate test results which are indicative of patient's status allows caregivers to quickly analyze and diagnose a patient, which leads to faster treatment and improved outcomes. We noted additional workflow benefits, for which the expert respiratory therapist did not have to leave a critical situation for approximately $10 \mathrm{~min}$ to run a blood gas sample. Rather they can remain an integral part of a team. Additional benefits that we observed, but did not quantify, were that POCT has accelerated decision-making, reduced re-draws, enabled rapid response to patients (e.g. ventilator weaning), and increased efficiency and productivity. The POCT system is user-friendly, which allows nonlaboratory professionals such as respiratory care practitioners to operate the instruments [2]. Our experience with the POCT system, reiterates that of others, who found staff engagement higher with satellite testing and POCT systems than with central lab testing [28]. The devices are self-contained, are low maintenance, and have on-screen instructions that promote ease of use. The devices we chose also use disposable test cartridges, which are readily replaced and enhances ease of use.
The one challenge we discovered during implementation was that we did not predict the education and training that was required of the team to perform point-of-care-testing reliably. As noted, mandatory and repetitive education occurred for the entire team. We also found that it took some focused effort to determine the most efficient method of ordering sampling cartridges. We know that the more cards you can purchase at one time, the less quality control needs to be completed; however, the cards expire and waste due to overstocking is not optimal. We now order cards twice annually.

Point-of-care blood gas testing has provided a more efficient process for the respiratory care practitioners as they no longer need to physically leave a patient room and travel with the specimen through patient care areas to run a sample in a bench top machine. Test results are accurate per our routine side-by-side comparison with the central laboratory, we believe there are fewer specimen errors due to elimination of transport, and the rapid turnaround time has possibly led to improved patient outcomes as seen in our sepsis data.

In today's climate, with health care systems dealing with the challenges of a global pandemic [35], the availability of POCT for blood gases has proved invaluable at our institution. As most hospitals around the world have experienced, we are caring for an unprecedented volume of acutely ill patients. We implemented detailed protocols to ensure staff safety and mitigate nosocomial spread of the disease, requiring staff to frequently don and doff personal protective equipment (PPE). POCT allows us to follow these stringent infection prevention protocols without delaying critical patient care. The POCT analyzers are brought into patient rooms that are COVID-19 positive. This circumvents the need for multiple cycles of robing/disrobing of PPE and additionally eliminates the need to transport contaminated blood through patient care areas. A POCT device can be stored in the ante room so that it is readily available for those patients that require frequent blood gases. Therefore, we believe that using POCT has allowed us to provide the same response time for critical assessment and treatment of patients with COVID-19 as with our non-COVID patients. To confirm, an analysis of lactate and sepsis bundle compliance using the POCT before and after the onset of the pandemic would be of value.

\section{LIMITATIONS}

Several limitations of this study should be addressed. The impact of implementing POCT testing at our institution was done retrospectively, so we could not eliminate the possibility of bias in our observations due to patient selection, policy changes, temporal trends, or other confounders that we could not control for prospectively. The analysis was descriptive in nature, so caution must be used in interpreting any causal relationship between implementation of POCT and improvements in sepsis bundle compliance or mortality rates in sepsis patients.

Due to the data collection, timelines, electronic medical record revisions, and other confounding factors, it was not possible for us to collect data over a consistent time period which would have been ideal. We also conducted the employee satisfaction survey one time after the intervention; however, the authors recognize that a controlled survey before and after implementation would have been ideal. We distributed the evaluation tool to all team members and hand selected only those that were here during the period where both analyzers were used, which may be viewed as bias and resulted in a small number of participants. We included $100 \%$ of team member surveys that had experience with both systems.

\section{CONCLUSION}

Our hospital changed from traditional benchtop analyzers for use with all blood gas analysis to a POCT system, and our experience with the new system has been positive. We have observed hospital cost savings, demonstrated improved employee engagement, and improved compliance with lactate turnaround time, which has improved sepsis bundle compliance and may be related to lower mortality rates. We have noted additional advantages of using the POCT system in conjunction with stringent infection control protocols. 


\section{DISCLOSURES}

\section{Acknowledgments}

We would like to thank Dana R Fletcher, PhD, for editing the manuscript.

\section{Contributors}

Study design and data acquisition, PAD; data analysis, RAC and PAD; literature search, APJ; manuscript preparation and review. PAD, JPH, RMK, and APJ. All authors have approved the final version.

\section{Funding}

This study did not receive any specific grant from funding agencies in the public, commercial, or not-for-profit sectors.

\section{Competing interests}

All authors have completed the ICMJE uniform disclosure form at www. icmje.org/coi_disclosure.pdf.

\section{Ethical approval}

Ethical Requirement of Research Ethics Board approval for this process improvement project was not required by the institution.

\section{REFERENCES}

1. Clifford L-J. The pros and cons of point-of-care testing vs laboratory testing. Med Lab Obs 2018: 34-8.

2. Louie RF, Tang Z, Shelby DG, Kost GJ. Point-of-care testing: millennium technology for critical care. Lab Med 2000; 31(7): 402-8. doi: 10.1309/0Y5F-B7NP-5Y67-GW7T

3. Nichols JH. Point of care testing. Clin Lab Med 2007; 27(4): 893-908. doi: 10.1016/j.cll.2007.07.003

4. Jang JY, Shin S Do, Lee EJ, Park CB, Song KJ, Singer AJ. Use of a comprehensive metabolic panel point-of-care test to reduce length of stay in the emergency department: a randomized controlled trial. Ann Emerg Med 2013; 61(2): 145-51. doi: 10.1016/j.annemergmed. 2012.07.021

5. Ward M, Self W, Singer A, Lazar D, Pines J. Cost-effectiveness analysis of early point-of-care lactate testing in the emergency department. J Crit Care 2016; 36: 69-75. doi: 10.1016/j.jcrc.2016.06.031

6. Goldstein LN, Wells M, Vincent-Lambert C. The cost-effectiveness of upfront point-of-care testing in the emergency department: a secondary analysis of a randomised, controlled trial. Scand J Trauma Resusc Emerg Med 2019; 27(1): 1-12. doi: 10.1186/s13049-019-0687-2

7. College of American Pathologists. Point-of-care testing toolkit. Northfield, IL, USA: College of American Pathologists; 2015.

8. Lee-Lewandrowski E, Lewandrowski K. Perspectives on cost and outcomes for point-of-care testing. Clin Lab Med 2009; 29(3): 479-89. doi: 10.1016/j.cll.2009.07.001

9. Kankaanpää M, Raitakari M, Muukkonen L, et al. Use of point-of-care testing and early assessment model reduces length of stay for ambulatory patients in an emergency department. Scand J Trauma Resusc Emerg Med 2016; 24(1): 1-7. doi: 10.1186/s13049-016-0319-z

10. Lee EJ, Shin S Do, Song KJ, et al. A point-of-care chemistry test for reduction of turnaround and clinical decision time. Am J Emerg Med 2011; 29(5): 489-95. doi: 10.1016/j.ajem.2009.11.020

11. Trans N, Kost G. Point-of-care testing in critical care medicine: improving outcomes, cost effectiveness and turnaround time. Int J Intensive Care 2003; 10(3): 131-4.

12. Giuliano KK, Grant ME. Blood analysis at the point of care: issues in application for use in critically ill patients. AACN Clin Issues 2002; 13(2): 204-20. doi: 10.1097/00044067-200205000-00006

13. John A St., C.P. P. Benefits of point-of-care testing in the emergency department. Clin Chem Lab Med 2015; 53(March): S142. Available at: http://ovidsp.ovid.com/ovidweb.cgi? $T=J S \& P A G E=$ reference $\& D=e m e d$ 13\&NEWS $=\mathrm{N} \& A \mathrm{AN}=71910430$

14. Kendall J, Reeves B, Clancy M. Point of care testing: randomised controlled trial of clinical outcome. Br Med J 1998; 316(7137): 1052-7. doi: 10.1136/bmj.316.7137.1052
15. Severinghaus JW, Astrup P, Murray JF. Blood gas analysis and critical care medicine. Am J Respir Crit Care Med 1998; 157(4 Pt 2): S114-22. doi: 10.1164/ajrccm.157.4.nhlb1-9

16. Zwisler ST, Zincuk Y, Bering CB, Zincuk A, Nybo M, Mikkelsen S. Diagnostic value of prehospital arterial blood gas measurements - a randomised controlled trial. Scand J Trauma Resusc Emerg Med 2019; 27(1): 1-9. doi: 10.1186/s13049-019-0612-8

17. Angus DC, van der Poll T. Severe sepsis and septic shock. N Engl J Med 2013; 369(9): 840-51. doi: 10.1056/NEJMra1208623

18. Dantes RB, Epstein L. Combatting sepsis: a public health perspective. Clin Infect Dis 2018; 67(8): 1300-2. doi: 10.1093/cid/ciy342

19. Lee SM, Kim SE, Bin KE, Jeong HJ, Son YK, An WS. Lactate clearance and vasopressor seem to be predictors for mortality in severe sepsis patients with lactic acidosis supplementing sodium bicarbonate: a retrospective analysis. PLoS One 2015; 10(12): 1-14. doi: 10.1371/journal. pone. 0145181

20. Lee SM, An WS. New clinical criteria for septic shock: serum lactate level as new emerging vital sign. J Thorac Dis 2016; 8(7): 1388-90. doi: $10.21037 /$ jtd.2016.05.55

21. Dellinger RP, Levy MM, Rhodes A, et al. Surviving sepsis campaign: international guidelines for management of severe sepsis and septic shock, 2012. Intensive Care Med 2013; 39(2): 165-228. doi: 10.1007/ s00134-012-2769-8

22. Shankar-Hari M, Phillips GS, Levy ML, et al. Developing a new definition and assessing new clinical criteria for septic shock: for the third international consensus definitions for sepsis and septic shock (sepsis-3). JAMA 2016; 315(8): 775-87. doi: 10.1001/jama.2016.0289

23. Broder G, Weil M. Excess lactate, an index of reversibility of shock in human patients. Science 1964; 143(3613): 1457-9. doi: 10.1126/ science.143.3613.1457

24. Asha SE, Chan ACF, Walter E, et al. Impact from point-of-care devices on emergency department patient processing times compared with central laboratory testing of blood samples: a randomised controlled trial and cost-effectiveness analysis. Emerg Med J 2014; 31(9): 714-19. doi: 10.1136/ emermed-2013-202632

25. Levy MM, Dellinger RP, Townsend SR, et al. The surviving sepsis campaign: results of an international guideline-based performance improvement program targeting severe sepsis. Intensive Care Med 2010; 36(2): 222-31. doi: 10.1007/s00134-009-1738-3

26. Kantartjis M, Melanson S. What is new in point-of-care testing? Point Care 2018; 17(1): 1-8. doi: 10.1097/POC.0000000000000157

27. Slavitt A. The COVID-19 pandemic underscores the need to address structural challenges of the US health care system. JAMA Heal Forum 2020; 1(7): e200839. doi: 10.1001/jamahealthforum.2020.0839

28. Kilgore ML, Steindel SJ, Smith JA. Evaluating stat testing options in an academic health center: therapeutic turnaround time and staff satisfaction. Clin Chem 1998; 44(8): 1597-603. doi: 10.1093/clinchem/44.8.1597

29. Kilgore ML, Steindel SJ, Smith JA. Cost analysis for decision support: the case of comparing centralized versus distributed methods for blood gas testing. J Healthc Manag 1999; 44(3): 207-16. doi: 10.1097/00115514-199905000-00011

30. Arnold RC, Shapiro NI, Jones AE, et al. Multicenter study of early lactate clearance as a determinant of survival in patients with presumed sepsis. Shock 2009; 32(1): 35-9. doi: 10.1097/SHK.0b013e3181971d47

31. Bakker J. Lactate is the target for early resuscitation in sepsis. Rev Bras Ter Intensiva 2017; 29(2): 124-7. doi: 10.5935/0103-507X.20170021

32. Mikkelsen ME, Miltiades AN, Gaieski DF, et al. Serum lactate is associated with mortality in severe sepsis independent of organ failure and shock. Crit Care Med 2009; 37(5): 1670-7. doi: 10.1097/ CCM.0b013e31819fcf68

33. Nguyen HB, Rivers EP, Knoblich BP, et al. Early lactate clearance is associated with improved outcome in severe sepsis and septic shock. Crit Care Med 2004; 32(8): 1637-42. doi: 10.1097/01.ccm.0000132904.35713.a7

34. Puskarich MA, Trzeciak S, Shapiro NI, Heffner AC, Kline JA, Jones AE. Outcomes of patients undergoing early sepsis resuscitation for cryptic shock compared with overt shock. Resuscitation 2011; 82(10): 1289-93. doi: 10.1016/j.resuscitation.2011.06.015

35. Blumenthal D, Fowler EJ, Abrams M, Collins SR. Covid-19 - implications for the health care system. N Engl J Med 2020; 383(15): 1483-8. doi: 10.1056/NEJMsb2021088 